\title{
A Switching Strategy for Robust Cooperative Exploration
}

\author{
Wencen $\mathrm{Wu}$ and Fumin Zhang
}

\begin{abstract}
This paper develops a switching strategy for networked mobile sensing agents to automatically decide when to perform individual exploration and when to perform cooperative exploration of an unknown scalar field corrupted by time-varying non-Gaussian noises. The switching condition from individual exploration to cooperative exploration is based on Razumikhin theorem, and the change of the signal-to-noise ratio (SNR) serves as the switching condition from cooperative exploration to individual exploration. A cooperative $H_{\infty}$ filter is proposed when the agents are collaborating. Sufficient conditions are derived for the cooperative $H_{\infty}$ filter to converge. This switching strategy balances the mobility of the agents and the exploring accuracy. Simulation results demonstrate that with the switching strategy, a group of sensing agents can succeed in finding a local minimum of an unknown scalar field efficiently.
\end{abstract}

\section{INTRODUCTION}

Cooperative exploration problems are formulated and investigated recently [1]-[4]. A cooperative network of sensing agents are expected to perform better than a single agent or a number of sensors that are fixed when accuracy and adaptiveness are concerned. However, a single agent has advantages when mobility and the cost of the system are concerned. In order to make the best use of resources, the exploring behavior for each sensing agent does not have to be fixed. Biologists have observed switching between individual and cooperative behaviors in nature. For example, it is conjectured that fish in a group communicate and exchange information with others only when they are not confident with the information gathered individually [5]. Some recent work on exploration and exploitation [6], [7] and task allocation [8], [9] are also related to switching strategies.

We consider the mission of searching for a local minimum of an unknown scalar field with multiple sensing agents. The field is assumed to be corrupted by time-varying nonGaussian noises. This is a challenging task, but it is closer to real world applications than the Gaussian noise assumption in most literature. A switching strategy for the networked sensing agents is developed so that each agent makes decision at every time step about whether to perform individual exploration or cooperative exploration. Inspired by the Razumikhin theorem [10] [11], which is widely used in justifying the stability of time-delay systems, we introduce sufficient conditions for the sensing agents to examine whether they will converge to a field minimum by conducting individual

The research is supported by ONR grants N00014-08-1-1007 and N00014-09-1-1074, and NSF grants ECCS-0841195, ECCS0845333(CAREER) and CNS-0931576. Wencen $\mathrm{Wu}$ and Fumin Zhang are with the School of Electrical and Computer Engineering, Georgia Institute of Technology, Savannah, GA 31407, USA wwencen3, fuminegatech.edu exploration. If the sufficient conditions are not violated, which means each agent can find the local minimum by itself, then an $H_{\infty}$ filter is running and producing filtered measurements of the field, which are used to give estimates of the field gradients. The estimated gradients are utilized so that by following the opposite directions of the gradients, the agents may find a local minimum of the field [12]. If the switching conditions of convergence are violated, the agents switch to cooperative exploration to obtain more accurate estimates of the gradients. A cooperative $H_{\infty}$ filter is constructed to give estimated field values and gradients at the formation center formed by the agents. During the cooperative exploration phase, if the agents detect that the signal-to-noise (SNR) ratio of the field increases by a sufficient amount, they switch back to individual exploration with the hope that convergence can be achieved. Our switching strategy in this paper is based on rigorous convergence analysis.

The $H_{\infty}$ filter differs from the Kalman filter in that it does not require the knowledge of the noise properties except that the noises are assumed to have bounded power. Note that the Kalman filter assumes the noises to be Gaussian [13] [15]. Therefore, the $H_{\infty}$ filter is robust to time-varying nonGaussian noises. An important constraint of the $H_{\infty}$ filter is that the existence of the filter requires the fulfillment of a set of feasibility conditions, which further posts constraints on the exploration behaviors for the individual agents and the cooperative agent formation. Convergence analysis of $H_{\infty}$ filters has been performed in [16] [17]. Based on these work, we develop sufficient conditions for the cooperative $H_{\infty}$ filter to admit feasible solutions and convergence.

This paper is organized as follows. In section II, we introduce the information dynamics and the searching strategy for an individual agent. Convergence analysis based on the Razumikhin Theorem is performed. We propose when to switch from individual exploration behavior to cooperative exploration behavior. In section III, a cooperative $H_{\infty}$ filter is constructed and the feasibility and convergence of the $H_{\infty}$ filter are investigated. We propose when to switch from cooperative exploration behavior back to individual exploration behavior. Simulation results are shown in section V. Concluding remarks are presented in section VI.

\section{INDIVIDUAL EXPLORATION}

\section{A. Information Dynamics and $H_{\infty}$ Filter}

Suppose that $z(\mathbf{r}), \mathbf{r} \in \mathbb{R}^{2}$ is an unknown smooth scalar field which is perturbed by time-varying non-Gaussian noises. Our goal is to locate the positions of the local minimums of the field. In this paper, we assume that the motion of each agent obeys first order dynamics. Extension 
to second order dynamics can be made without changing the switching strategy and the $H_{\infty}$ filtering results.

Denote the position of a sensing agent at the $k$ th step as $\mathbf{r}_{k}$, the measurement taken by the agent as $p_{k}$ and the true field value at position $\mathbf{r}_{k}$ as $z_{k}$. When the noise level is low, the sensing agent can perform individual exploration, making use of the successive measurements taken along its trajectory. In this case, we define the state to be the field value: $s_{k}=z_{k}$. Using the Taylor's expansion, the state equation is

$$
s_{k+1}=s_{k}+\left(\mathbf{r}_{k+1}-\mathbf{r}_{k}\right)^{T} \nabla z_{k}+w_{k},
$$

and the measurement equation is

$$
p_{k}=s_{k}+v_{k},
$$

where $\nabla z_{k}$ is the gradient of the field at position $\mathbf{r}_{k}$ and $w_{k}$ and $v_{k}$ represent the state noise and sensor noise. We assume that $w_{k}$ and $v_{k}$ have bounded power, but other properties are unknown.

Given the state equation (1) and the measurement equation (2), an $H_{\infty}$ filter can be computed by the agent. The goal of the $H_{\infty}$ filter is to guarantee that the ratio between the energy of the estimation error and the energy of the disturbances is less than a prescribed attenuation level $\gamma$, which can be expressed as $J<\gamma^{2}$ where the cost function $J$ of the agent is defined as

$$
J=\frac{\sum_{k=0}^{M-1}\left\|s_{k}-\hat{s}_{k}\right\|_{Q_{k}}^{2}}{\left\|s_{0}-\hat{s}_{0}\right\|_{P_{0}^{-1}}^{2}+\sum_{k=0}^{M-1}\left(\left\|w_{k}\right\|_{W_{k}^{-1}}^{2}+\left\|v_{k}\right\|_{V_{k}^{-1}}^{2}\right)} .
$$

In the cost function, $\hat{s}_{0}$ is the initial estimate of $s_{0}, P_{0}>$ $0, Q_{k} \geq 0, W_{k}>0$ and $V_{k}>0$ are the weighting matrices chosen by the designer, which depend on the noise strengths. For example, $W_{k}>V_{k}$ if we know that the sensor noise is stronger than the state noise.

Following the general steps of constructing the $H_{\infty}$ filter in [13], we can write down the $H_{\infty}$ filter equations as

$$
\begin{aligned}
& S_{k}=P_{k}^{-1}-\frac{1}{\gamma^{2}} Q_{k}+V_{k}^{-1}, \\
& K_{k}=S_{k}^{-1} V_{k}^{-1}, \\
& \hat{s}_{k+1}=\hat{s}_{k}+\left(\mathbf{r}_{k+1}-\mathbf{r}_{k}\right)^{T} \nabla \hat{z}_{k}+K_{k}\left(p_{k}-\hat{s}_{k}\right), \\
& P_{k+1}=S_{k}^{-1}+W_{k},
\end{aligned}
$$

where $\nabla \hat{z}_{k}$ is the gradient of the field estimated by the agent. The output of the $H_{\infty}$ filter is the filtered value $\hat{s}_{k}$.

\section{B. Exploration Behavior for Single Sensing Agents}

To search for a local minimum, the agent moves in directions that may reduce the field value. For the sake of simplicity, we assume the agent moves in the opposite direction of the gradient in this paper. Therefore, the dynamics can be expressed as

$$
\dot{\mathbf{r}}_{k}=-\nabla \hat{z}_{k},
$$

Note that equation (5) is not the only strategy that may achieve a local minimum. It is well known that biological entities such as the E. Coli switches between tumbling motion and straight line motion for gradient climbing [18]. We will see that the switching strategy developed in this paper does not depend on specific searching behaviors.

At each time step $k$, the agent estimates the gradient of the field $\nabla z_{k}$ at the current position using the current and previous measurements. If $\mathbf{r}_{k}-\mathbf{r}_{k-1}=\mathbf{r}_{k-1}-\mathbf{r}_{k-2}$, then the agent may move along a straight line. In this case, we have

$$
\nabla \hat{z}_{k}=\frac{\hat{s}_{k}-\hat{s}_{k-1}}{\left\|\mathbf{r}_{k}-\mathbf{r}_{k-1}\right\|}\left(\mathbf{r}_{k}-\mathbf{r}_{k-1}\right)+\delta
$$

where $\delta$ is a small perturbation that prevents the agent from keeping moving along the same straight line. Otherwise, note that $D_{h} z_{k}=\nabla z_{k} \cdot h$ where $D_{h} z_{k}$ is the directional derivative of the field value at position $\mathbf{r}_{k}$ in the direction $h$. If the successive positions of the agent are close enough, the gradient at position $\mathbf{r}_{k}$ can be approximated by solving the following two (in 2D) equations. In 3D, there will be three equations, which involve steps $k, k-1$ and $k-2$.

$$
\begin{aligned}
& \frac{\hat{s}_{k}-\hat{s}_{k-1}}{\left\|\mathbf{r}_{k}-\mathbf{r}_{k-1}\right\|}=\nabla \hat{z}_{k} \cdot\left(\mathbf{r}_{k}-\mathbf{r}_{k-1}\right), \\
& \frac{\hat{s}_{k-1}-\hat{s}_{k-2}}{\left\|\mathbf{r}_{k-1}-\mathbf{r}_{k-2}\right\|}=\nabla \hat{z}_{k} \cdot\left(\mathbf{r}_{k-1}-\mathbf{r}_{k-2}\right) .
\end{aligned}
$$

Since there are noises in the field and the measuring process, the estimated gradient may be inaccurate when the noise level is high, which may lead to the failure of the searching behavior. Thus, we need to figure out the conditions under which the agent will converge to the field minimum if it keeps searching the field independently. Taking advantage of the Razumikhin Theorem and the related work in discrete time-delay systems, we propose the following exploration strategy for a agent to make decisions on when to switch to collaboration.

Algorithm 1: Suppose a sensing agent is searching for a local minimum of an unknown field where the field value satisfies $z_{k} \geq 0$. Define a continuous nondecreasing function $w:[0, \infty) \rightarrow[0, \infty)$ with $w(u)>0$ if $u>0$ and $w(0)=0$. Let $\bar{s}_{k}=\max _{s \in[-r, 0]} \hat{s}_{k+s}$.

At each time step $k \geq r$ where $r$ is a positive constant,

(1) The agent takes a measurement of the field and runs an $H_{\infty}$ filter. The output of the $H_{\infty}$ filter is $\hat{s}_{k}$. Then the estimate of the field gradient $\nabla \hat{z}_{k}$ can be computed using equations (7) and (8).

(2) The agent moves in the opposite direction of the estimated gradient $\dot{\mathbf{r}}_{k}=-\nabla \hat{z}_{k}$ or uses other strategies to reduce the measured field value. Then the agent gets a new filtered value $\hat{s}_{k+1}$,

(3) If $K \hat{s}_{k} \geq \bar{s}_{k}$ where $K>1$ is a constant, the agent checks the value of $\hat{s}_{k+1}-\hat{s}_{k}$. If $\hat{s}_{k+1}-\hat{s}_{k} \leq-w\left(\left\|\hat{s}_{k}\right\|\right)$, then the agent keeps exploring the field independently. Otherwise, the agent switches to cooperative exploration behavior.

To justify the algorithm and the switching condition, we first restate the Razumikhin Theorem for the asymptotic stability of time-delay systems [10] without proof.

Theorem 1: (Razumikhin Theorem) Given a system $\dot{x}(t)=f\left(t, x_{t}\right)$ where $x \in \mathbb{R}^{n}$ and $x_{t}$ represents the delayed system trajectory, suppose $f: \mathbb{R} \times C \rightarrow \mathbb{R}^{n}$ takes bounded 
subsets of $C$ into bounded subsets of $\mathbb{R}^{n}$. Suppose $\alpha_{1}, \alpha_{2}, w$ : $\mathbb{R}_{+} \rightarrow \mathbb{R}_{+}$are continuous nondecreasing functions, $\alpha_{1}(u)>$ $0, \alpha_{2}(u)>0$ and $w(u)>0$ for $u>0$, and $\alpha_{1}(0)=\alpha_{2}(0)=0$, $\alpha_{2}$ strictly increasing. Suppose there exists a continuous nondecreasing function $g(u)>u$ for $u>0$. If there exists a continuous differentiable function $V: \mathbb{R} \times \mathbb{R}^{n} \rightarrow \mathbb{R}$ such that

$$
\alpha_{1}(\|x\|) \leq V(t, x) \leq \alpha_{2}(\|x\|), \forall t \in \mathbb{R}, x \in \mathbb{R}^{n},
$$

and the derivative of $V$ along the solution $x(t)$ satisfies

$$
\dot{V}(t, x(t)) \leq-w(\|x(t)\|),
$$

whenever $V(t+\theta), x(t+\theta)) \leq g(V(t, x(t)))$ for all $\theta \in[-r, 0]$, then the equilibrium $x(t)=0$ of the system is asymptotically stable.

In discrete systems, condition (10) becomes [11]

$$
V(k+1, x(k+1))-V(k, x(k)) \leq-w(\|x(k)\|),
$$

whenever $V(k+\theta), x(k+\theta)) \leq g(V(k, x(k)))$ for all $\theta \in$ $[-r, 0]$.

Based on the Razumikhin Theorem, we propose the following corollary that justifies the switching condition (3) in algorithm 1.

Corollary 1: Suppose the field value $z_{k}$ satisfies $z_{k} \geq 0$. Let $\bar{s}_{k}=\max _{s \in[-r, 0]} \hat{s}_{k+s}$ where $r \in \mathbf{Z}^{+}$and $\hat{s}_{k}$ is the output of the $H_{\infty}$ filter. Define continuous nondecreasing functions $w:[0, \infty) \rightarrow[0, \infty)$ with $w(u)>0$ if $u>0$ and $w(0)=0$. If $\hat{s}_{k+1}-\hat{s}_{k} \leq-w\left(\left\|\hat{s}_{k}\right\|\right)$ whenever $K \hat{s}_{k} \geq \bar{s}_{k}$ where $K>1$ is a constant, then $\hat{s}_{k}$ will converge to 0 as $k \rightarrow \infty$.

Proof: Let $V\left(\hat{s}_{k}\right)=\hat{s}_{k} \geq 0$. Choose $g\left(V\left(\hat{s}_{k}\right)\right)=K \hat{s}_{k}$. Then the condition $V\left(\bar{s}_{k}\right) \leq g\left(V\left(\hat{s}_{k}\right)\right)$ becomes $\bar{s}_{k} \leq K \hat{s}_{k}$. We also have $V\left(\hat{s}_{k+1}\right)-V\left(\hat{s}_{k}\right)=\hat{s}_{k+1}-\hat{s}_{k}$. Therefore, according to the Razumikhin Theorem, if for all $k \in[r, \infty)$, the measurement satisfies $\hat{s}_{k+1}-\hat{s}_{k} \leq-w\left(\left\|\hat{s}_{k}\right\|\right)$ whenever $K \hat{s}_{k} \geq \bar{s}_{k}$, then $\hat{s}_{k}$ converges to 0 as $k \rightarrow \infty$. Since $H_{\infty}$ filter is unbiased [14] and $\hat{s}_{k}$ is the output of the $H_{\infty}$ filter, the agent will converge to the minimum point of the field.

The switching strategy (3) requires the agents to cooperate when the sufficient conditions for convergence in the Corollary are violated. Note that violation of the sufficient conditions does NOT necessarily lead to failure of convergence. However, it can be seen that as the time window $r$ becomes sufficiently large, since $V$ is corrupted by noises, violation of the sufficient conditions will lead to slow convergence speed as will be seen in the simulation results. The window size $r$ is therefore an important parameter in our algorithm. More in-depth results on learning appropriate value of " $r$ " through adaptive strategies are under investigation.

\section{CoOperative Exploration Strategy}

When the sensing agents detect that the conditions in Algorithm 1 are violated, which implies that the noise level of the field may be too high to acquire the gradient information of the field, they start to communicate with each other and switch to cooperative exploration. For the sake of simplicity, we here assume that an agent is able to communicate with all other agents when necessary. If this assumption does not hold and the communication capability is constrained by distances, the cooperative filtering techniques will only involve agents that can communicate with each other. Maintaining connectivity is not a trivial task [19], but it is not the focus of this paper.

\section{A. Information Dynamics and the Cooperative $H_{\infty}$ Filter}

In the cooperative exploration phase, we define the information dynamics as described in [2] for a group of agents. Suppose we have $N$ sensing agents. The position of each agent at the $k$ th step is denoted as $\mathbf{r}_{i, k}$ where $i=1, \cdots, N$, the measurement taken by the $i$ th agent is $p_{i, k}$ and the true field value at position $\mathbf{r}_{i, k}$ is $z_{i, k}$. Denote the position of the formation center at the $k$ th step as $\mathbf{r}_{c, k}$ and the field value of the position center as $z_{c, k}$. Using Taylor's expansion to approximate $z_{i, k}$, we can get:

$z_{i, k} \approx z_{c, k}+\left(\mathbf{r}_{i, k}-\mathbf{r}_{c, k}\right)^{T} \nabla z_{c, k}+\frac{1}{2}\left(\mathbf{r}_{i, k}-\mathbf{r}_{c, k}\right)^{T} \nabla^{2} z_{c, k}\left(\mathbf{r}_{i, k}-\mathbf{r}_{c, k}\right)$.

where $\nabla z_{c, k}$ is the gradient of the field and $\nabla^{2} z_{c, k}$ is the Hessian of the field.

Define $\mathbf{h}_{k-1}=\left(0, E\left[H_{c, k}\left(\mathbf{r}_{c, k}-\mathbf{r}_{c, k-1}\right)\right]^{T}\right)^{T}$ and $A_{k}=$ $\left(\begin{array}{cc}1 & \left(\mathbf{r}_{c, k}-\mathbf{r}_{c, k-1}\right)^{T} \\ 0 & \mathbf{I}_{3 \times 3}\end{array}\right)$, where $H_{c, k}$ is the estimate of the Hessian $\nabla^{2} z_{c, k}$. We can see that $A_{k}$ is nonsingular. Choose the state to be $\mathbf{s}_{k}=\left(z_{c, k}, \nabla z_{c, k}^{T}\right)^{T}$, then the state equation can be expressed as

$$
\mathbf{s}_{k+1}=A_{k} \mathbf{s}_{k}+\mathbf{h}_{k}+\mathbf{w}_{k},
$$

where $\mathbf{w}_{k}$ is a $3 \times 1$ state noise vector

$$
\text { Let } C_{k}=\left(\begin{array}{cc}
1 & \left(\mathbf{r}_{1, k}-\mathbf{r}_{c, k}\right)^{T} \\
\vdots & \vdots \\
1 & \left(\mathbf{r}_{N, k}-\mathbf{r}_{c, k}\right)^{T}
\end{array}\right) \text { and } D_{k} \text { be the } N \times 4
$$

matrix with the $i$ th row vector defined by the Kronecker product $\frac{1}{2}\left(\left(\mathbf{r}_{i, k}-\mathbf{r}_{c, k}\right) \otimes\left(\mathbf{r}_{i, k}-\mathbf{r}_{c, k}\right)\right)^{T}$. Define a column vector $\vec{H}$ that represents the rearranged Hessian matrix $H_{c, k}$. For example, in $2 \mathrm{D}, \vec{H}=\left[\begin{array}{llll}H_{11} & H_{12} & H_{21} & H_{22}\end{array}\right]^{T}$. Then the measurement equation is:

$$
\mathbf{p}_{k}=C_{k} \mathbf{s}_{k}+D_{k} \vec{H}_{c, k}+\mathbf{v}_{k}
$$

where $\mathbf{v}_{k}$ is a $N \times 1$ measurement noise vector. We also assume that $\mathbf{w}_{k}$ and $\mathbf{v}_{k}$ have bounded power and, but other properties are unknown.

Given the state equation (13) and measurement equation (14), a cooperative $H_{\infty}$ filter is constructed, which generates estimates of the field value $\hat{z}_{c, k}$ and the field gradient $\nabla \hat{z}_{c, k}$ at each step. With a similar cost function $J$ defined in single agent case, the cooperative $H_{\infty}$ filter equations are:

$$
\begin{aligned}
& S_{k}=P_{k}^{-1}-\frac{1}{\gamma^{2}} Q_{k}+C_{k}^{T} V_{k}^{-1} C_{k}, \\
& K_{k}=S_{k}^{-1} C_{k}^{T} V_{k}^{-1}, \\
& \hat{s}_{k+1}=A_{k} \hat{s}_{k}+\mathbf{h}_{k}+A_{k} K_{k}\left(\mathbf{p}_{k}-C_{k} \hat{s}_{k}-D_{k} H_{k}\right), \\
& P_{k+1}=A_{k} S_{k}^{-1} A_{k}^{T}+W_{k},
\end{aligned}
$$

where $\mathbf{h}_{k}$ can be estimated using the technique described in [2] [20], which is based on curvature estimation. 
The center of the formation can be controlled to move as a single agent. For simplicity, we also direct the center to follow the opposite direction of the gradient direction: $\dot{\mathbf{r}}_{c, k}=-\nabla \hat{z}_{c, k}$.

\section{B. Convergence of the Cooperative $H_{\infty}$ Filter}

The $H_{\infty}$ filtering convergence analysis has been widely investigated in both continuous-time and discrete-time, e.g. in [14], [15], [21], [22] and the references therein. The main feasibility results in discrete-time are summarized here.

Theorem 2: Consider the system (13), (14) and the cost function (3). Under the condition that $A_{k}$ is nonsingular, an $H_{\infty}$ filter guaranteeing an attenuation level $\gamma$ exists if and only if there exist two sequences of positive definite matrices $\left\{S_{k}\right\}_{k=0}^{M-1}$ and $\left\{P_{k}\right\}_{k=0}^{M-1}$ such that

$$
\begin{aligned}
P_{k+1} & =A_{k} S_{k}^{-1} A_{k}^{T}+W_{k}, \\
S_{k} & =P_{k}^{-1}-\frac{1}{\gamma^{2}} Q_{k}+C_{k}^{T} V_{k}^{-1} C_{k}, \\
S_{0} & =P_{0}^{-1}, \\
S_{k} & >0, k=0,1, \cdots, M-1 .
\end{aligned}
$$

A feasible solution is defined as a positive definite solution $P_{k}$ of equation (16) that satisfies equation (17).

The difference Riccati equation (DRE) (16) can also be written as

$P_{k+1}=A_{k} P_{k} A_{k}^{T}-A_{k} P_{k}\left[\left(C_{k}^{T} V_{k}^{-1} C_{k}-\frac{1}{\gamma^{2}} Q_{k}\right)^{-1}+P_{k}\right]^{-1} P_{k} A_{k}^{T}+W_{k}$

Since the noise properties of the field are unknown, we can select $Q_{k} \rightarrow \sigma_{1}^{2} I, W_{k} \rightarrow \sigma_{2}^{2} I$ and $V_{k} \rightarrow \sigma_{3}^{2} I$ where $I$ is the identity matrix. As $k \rightarrow \infty$, if we drop the subscript $\infty$ for simplicity, then the Riccati equation (20) becomes

$$
P=A P A^{T}-A P\left[\left(\sigma_{3}^{-2} C^{T} C-\sigma_{1}^{2} \gamma^{-2} I\right)^{-1}+P\right]^{-1} P A^{T}+\sigma_{2}^{2} I .
$$

The finite-horizon $H_{\infty}$ problem becomes an infinite-horizon problem. If the solution to the infinite-horizon $H_{\infty}$ filter exists, then equation (21) admits a positive definite stabilizing solution $P^{s}$.

Also, when $k \rightarrow \infty$, the formation is stabilized, then $C$ goes to a constant matrix

$$
C=\left(\begin{array}{cc}
1 & \left(\mathbf{r}_{1}-\mathbf{r}_{c}\right)^{T} \\
\vdots & \vdots \\
1 & \left(\mathbf{r}_{N}-\mathbf{r}_{c}\right)^{T}
\end{array}\right)=\left(\begin{array}{cc}
1 & \mathbf{d}_{1}^{T} \\
\vdots & \vdots \\
1 & \mathbf{d}_{n}^{T}
\end{array}\right)
$$

In two dimensional space, $\mathbf{d}_{i}=\left[\begin{array}{ll}d_{i 1} & d_{i 2}\end{array}\right]^{T}$. In three dimensional space, $\mathbf{d}_{i}=\left[\begin{array}{lll}d_{i 1} & d_{i 2} & d_{i 3}\end{array}\right]^{T}$. We now apply the feasibility and convergence conditions to the cooperative $H_{\infty}$ filter and derive the sufficient conditions for the attenuation level and initial uncertainty so that the cooperative $H_{\infty}$ filter will converge. We have the following proposition.

Proposition 1: The solution $P_{k}$ of the Riccati equation (16) at every step $k$ is feasible and converges to the stabilizing solution $P^{s}$ when $k \rightarrow \infty$ if
(1) the attenuation level $\gamma$ satisfies:

$$
\begin{gathered}
\gamma^{2}>\max \left(0, \frac{\sigma_{1}^{2} \sigma_{3}^{2}}{N-\left|\sum_{i=1}^{N} d_{i 1}\right|-\left|\sum_{i=1}^{N} d_{i 2}\right|},\right. \\
\frac{\sigma_{1}^{2} \sigma_{3}^{2}}{\sum_{i=1}^{N} d_{i 1}^{2}-\left|\sum_{i=1}^{N} d_{i 1}\right|-\left|\sum_{i=1}^{N} d_{i 1} d_{i 2}\right|}, \\
\left.\frac{\sigma_{1}^{2} \sigma_{3}^{2}}{\sum_{i=1}^{N} d_{i 2}^{2}-\left|\sum_{i=1}^{N} d_{i 2}\right|-\left|\sum_{i=1}^{N} d_{i 1} d_{i 2}\right|}\right) .
\end{gathered}
$$

(2) $0<P_{0}<P^{s}$ where $P^{s}=\frac{1}{2}\left(\sigma_{2}^{2} I+\left(\sigma_{2}^{4} I+4 \sigma_{2}^{2} X\right)^{\frac{1}{2}}\right)$ and $X=\left(\sigma_{3}^{-2} C^{T} C-\sigma_{1}^{2} \gamma^{-2} I\right)^{-1}$.

Proof: To prove the proposition, we first consider the steady state solution $P^{s}$ of the Riccati equation (21). As $k \rightarrow \infty,\left(\mathbf{r}_{c, k+1}-\mathbf{r}_{c, k}\right)^{T}=0$. We can approximate $A_{k}$ by the identity matrix $I$. If we plug $A=I$ into equation (21), after rearranging, we can obtain

$$
P^{2}-\sigma_{2}^{2} P-\sigma_{2}^{2}\left(\sigma_{3}^{-2} C^{T} C-\sigma_{1}^{2} \gamma^{-2} I\right)^{-1}=0
$$

Define $X=\left(\sigma_{3}^{-2} C^{T} C-\sigma_{1}^{2} \gamma^{-2} I\right)^{-1}$. Then the above equation can be written as $P^{2}-\sigma_{2}^{2} P-\sigma_{2}^{2} X=0$. For a quadratic matrix equation of the form

$$
Q(Z)=A^{\prime} Z^{2}+B^{\prime} Z+C^{\prime}=0, A^{\prime}, B^{\prime}, C^{\prime} \in R^{n \times n},
$$

only when (1) $A^{\prime}=I$; (2) $B^{\prime}$ and $C^{\prime}$ commute; and (3) the square root of $B^{\prime 2}-4 C^{\prime}$ exists, we can apply the formula for the roots of a scalar quadratic equation and find a closed loop solution to this equation [23], which is $Z=\frac{1}{2}\left(-B^{\prime} \pm\right.$ $\left.\left(B^{\prime 2}-4 C^{\prime}\right)^{\frac{1}{2}}\right)$. By comparison, $A^{\prime}=I, B^{\prime}=-\sigma_{2}^{2} I$ and $C^{\prime}=$ $-\sigma_{2}^{2} X$ satisfy the first two conditions. If the square root of $\sigma_{2}^{4} I+4 \sigma_{2}^{2} X$ exists, then we can get the solution as $P^{s}=$ $\frac{1}{2}\left(\sigma_{2}^{2} I+\left(\sigma_{2}^{4} I+4 \sigma_{2}^{2} X\right)^{\frac{1}{2}}\right)$.

To check whether the square root of $\sigma_{2}^{4} I+4 \sigma_{2}^{2} X$ exists or not is equivalent to check whether $\sigma_{2}^{4} I+4 \sigma_{2}^{2} X$ is positive definite or not. From the facts that the identity matrix is positive definite and the sum of two positive definite matrices is positive definite, it is suffice to check the definiteness of $X^{-1}=\sigma_{3}^{-2} C^{T} C-\sigma_{1}^{2} \gamma^{-2} I$. We can compute that $C^{T} C=$ $\left(\begin{array}{cc}N & \sum_{i}^{N} \mathbf{d}_{i}^{T} \\ \sum_{i}^{N} \mathbf{d}_{i} & \sum_{i}^{N} \mathbf{d}_{i} \mathbf{d}_{i}^{T}\end{array}\right)$. Plug $C^{T} C$ into $X^{-1}$, we have

$$
X^{-1}=\left(\begin{array}{cc}
\sigma_{3}^{-2} N-\sigma_{1}^{2} \gamma^{-2} & \sigma_{3}^{-2} \sum_{i}^{N} \mathbf{d}_{i}^{T} \\
\sigma_{3}^{-2} \sum_{i}^{N} \mathbf{d}_{i} & \sigma_{3}^{-2} \sum_{i=1}^{N} \mathbf{d}_{i} \mathbf{d}_{i}^{T}-\sigma_{1}^{2} \gamma^{-2} I
\end{array}\right)
$$

We know that a symmetric matrix is positive definite if: (1) all the diagonal entries are positive, and (2) each diagonal entry is greater than the sum of the absolute values of all other entries in the same row. Therefore, we should have

$$
\sigma_{3}^{-2} \sum_{i=1}^{N} d_{i j}^{2}-\sigma_{1}^{2} \gamma^{-2}>\sigma_{3}^{-2}\left|\sum_{i=1}^{N} d_{i j}\right|+\sigma_{3}^{-2}\left|\sum_{i=1}^{N} d_{i 1} d_{i 2}\right|, j=1,2 .
$$

and

$$
\sigma_{3}^{-2} N-\sigma_{1}^{2} \gamma^{-2}>\sigma_{3}^{-2}\left|\sum_{i=1}^{N} d_{i 1}\right|+\sigma_{3}^{-2}\left|\sum_{i=1}^{N} d_{i 2}\right|
$$


which give us

$$
\gamma^{2}>\frac{\sigma_{1}^{2} \sigma_{3}^{2}}{N-\left|\sum_{i=1}^{N} d_{i 1}\right|-\left|\sum_{i=1}^{N} d_{i 2}\right|}>0,
$$

and

$$
\gamma^{2}>\frac{\sigma_{1}^{2} \sigma_{3}^{2}}{\sum_{i=1}^{N} d_{i j}^{2}-\left|\sum_{i=1}^{N} d_{i j}\right|-\left|\sum_{i=1}^{N} d_{i 1} d_{i 2}\right|}>0, j=1,2 .
$$

Therefore, the matrix $X^{-1}>0$ if the equation (23) is satisfied. This is the sufficient condition in our case that we can get the closed loop solution $P^{s}$. From Theorem 2 in [16], for some $\varepsilon>0$ and the solution $Y$ of a Lyapunov equation defined in [16], if $0<P_{0}<P^{s}+(Y+\varepsilon I)^{-1}$, then the solution $P_{k}$ of equation (16) is feasible $\forall k>0$ and converges to the stabilizing solution $P^{s}$ as $k \rightarrow \infty$. Since the matrix $Y$ is positive definite, we can consider a stricter condition, which is $0<P_{0}<P^{s}$. Therefore, if $0<P_{0}<P^{s}$, the solution $P_{k}$ to the Riccati equation (16) is feasible $\forall k$ and converges to the stabilizing solution $P^{s}$ as $k \rightarrow \infty$.

We can see from the proposition that we can achieve a lower noise attenuation level $\gamma$ and a smaller error bound $P^{s}$ as the number of agents increases and the formation gets larger. $\gamma$ and $P^{s}$ also depend on the noise strength in the field and the measurement process.

\section{Exploration Behavior for the Cooperative Sensing Agents}

One reason for the collaborating sensing agents to be supposed to perform better than a single agent is that at each step, the center of the formation takes the averaged filtered measurements from $N$ agents, which serves as an effective way of noise reduction, while a single agent can only make use of the time-series measurements in presence of noises. However, since we assume that the field is time-varying, in areas that the noise level reduces to the extent that an single agent is able to generate accurate gradient estimates, the cooperative sensing agents can break out the formation and start individual exploration again. Since each agent takes one measurement at a time, we define the following signalto-noise ratio that is based on the past $r$ measurements, where $r$ is the window size utilized in algorithm 1 .

$$
\beta_{i, k}=10 \lg \sum_{\xi=0}^{r} \frac{\hat{s}_{i, k-\xi}^{2}}{\left(p_{i, k-\xi}-\hat{s}_{i, k-\xi}\right)^{2}}, i=1, \cdots, N
$$

where $\hat{s}_{i, k-\xi}^{2}$ is the filtered field value computed by the $i$ th agent. The agents keeps track of the signal-to-noise ratio in cooperative exploration phase in order to detect the change of the field noise and evaluate whether the collaboration is worth keeping. We propose the following switching strategy for the cooperative sensing agents to decide when to switch back to individual exploration.

Algorithm 2: Define the average signal-to-noise ratio at time step $k$ as $\bar{\beta}_{k}=\frac{1}{N} \sum_{i=1}^{N} \beta_{i, k}$. Suppose that at time $T_{s}$, the sensing agents switch to cooperative exploration. Then for $k>T_{s}+r$, the cooperative sensing agents switch back to individual exploration if $\bar{\beta}_{k}>\mu \bar{\beta}_{T_{s}+r}$ where $\mu>1$ is a constant.
The agents only compute $\beta_{i, k}$ while they are performing cooperative exploration since the estimates of the signal-tonoise ratio when they are searching the field individually with only one measurement per step are usually not accurate. In the algorithm, $\bar{\beta}_{T_{S}+r}$ is the average signal-to-noise ratio after the agents switched to cooperative exploration, which implies that the agents may not be able to locate the field minimum under this noise level. $\mu$ is a constant chosen by the design which is based on the experience of the performance of the agents. After switch back to individual exploration, agents start to check switching conditions in algorithm 1 again.

\section{Simulation Results}

To testify our switching exploration strategy, we simulate four sensing agents searching for a minimum located in the center of a two dimensional scalar field which is corrupted by time-varying uniformly distributed noises. The field equation is $z=(x-10)^{2}+2(y-10)^{2}$. We assume that at time step $k=80$, the noise in the field increases from $5 \%$ to $30 \%$ and at time step $k=200$, the noise in the field reduces from $30 \%$ to $5 \%$. We choose $r=20, w=0.2 \hat{s}_{k}$ and $K=1.1$ in algorithm 1 and $\mu=1.35$ in algorithm 2 .

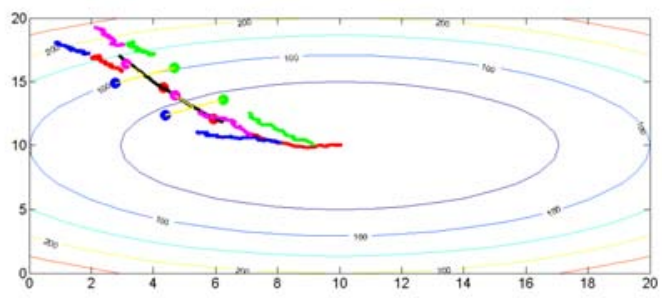

Fig. 1. The trajectories of the sensing agents. The four agents begin with individual exploration, then switch to cooperative exploration. At last they switch back to individual exploration and converge to the minimum of the field.

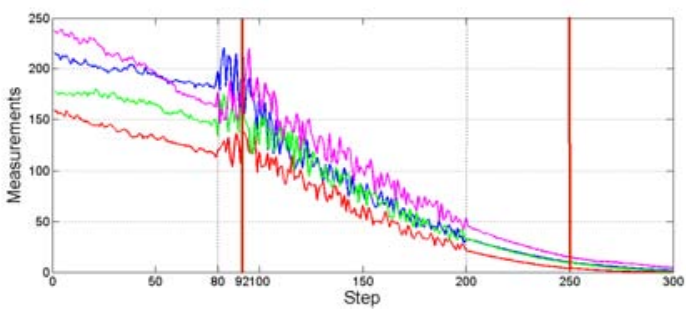

Fig. 2. Measurements taken by the agents. The agents switch to cooperative exploration at $k=92$ and switch back to individual exploration at $k=250$.

Figure 1 illustrates the exploration process of four sensing agents. The colored lines are trajectories of the four agents when they are performing individual exploration. The black line is the trajectory of the formation center when the agents are collaborating. The formation is controlled to be symmetric using the formation control technique described in [24]. Figure 2 shows the filtered field values measured by each agent with different colored lines corresponding to different agents in figure 1 . From figure 2, we can see that the four sensing agents start with individual exploration. At 


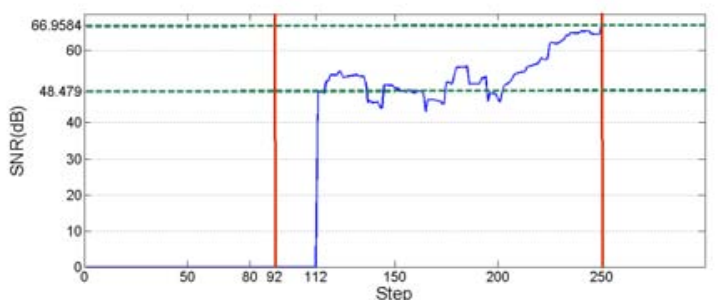

Fig. 3. The average signal-to-noise ratio. When $k<92$ and $k>250$, the agents perform individual exploration without computing $\beta_{k}$. At $k=250$, they switch back to individual exploration.

$k=92$ as indicated by the first solid red line in figure 2, they detect that the condition $\hat{s}_{k+1}-\hat{s}_{k} \leq-0.2 \hat{s}_{k}$ whenever $1.1 \hat{s}_{k+1} \geq \bar{s}_{k}$ is violated, then they switch to cooperative exploration. Therefore, $T_{s}=92$. When $k>T_{s}+r$ the agents begin to calculate the average signal-to-noise ratio $\bar{\beta}_{k}$ using the past $r$ measurements. $\beta_{T_{s}+r}=\beta_{92+20}=48.4790$. When $k>200$, the noise level in the field is decreased, which is indicated in figure 2 by the second solid red line. At $k=250$, the agents detect that $\bar{\beta}_{250}=66.9584$, which means that $\bar{\beta}_{k}>1.35 \bar{\beta}_{T_{s}+r}$ is satisfied. Then they switch back to cooperative exploration and at last converge to the minimum of the field.

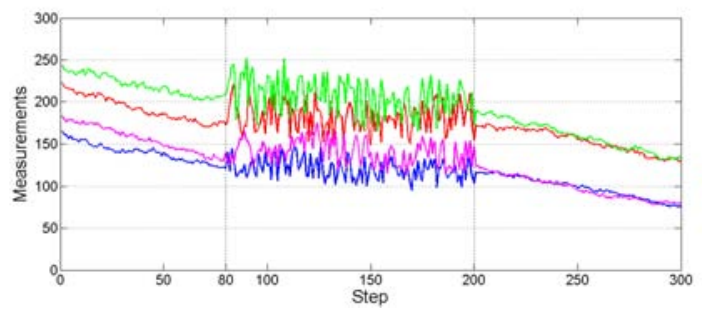

Fig. 4. Field values taken by the agents in the same setting as in figure 1 but without switching strategy.

For comparison, we also simulate four agents exploring the same field individually without the switching strategy under the same settings. Figure 4 shows the measurements taken by the four agents. From the figure, we can see that after 300 steps, the four agents still don't converge to the minimum. From the simulation results, we can tell that the algorithms developed in this paper can make the sensing agents perform better and converge faster to the minimum of the field.

\section{CONClusion AND Future WORK}

We develop a switching strategy for a group of sensing agents to search for local minimums of an unknown scalar field corrupted by time-varying non-Gaussian noises. Based on the Razumikhin theorem, we propose algorithms for decision making of each agent to decide whether to switch to cooperative exploration. The switching from cooperative exploration to individual exploration is based on the change of the signal-to-noise ratio of the field. A cooperative $H_{\infty}$ filter is constructed to give estimates of the field value and the gradient. We also rigorously justify the convergence and feasibility of the cooperative $H_{\infty}$ filter.

\section{REFERENCES}

[1] P. Ogren, E. Fiorelli, and N. E. Leonard, "Cooperative control of mobile sensor networks: Adaptive gradient climbing in a distributed environment," IEEE Transactions on Automatic Control, vol. 49, no. 8, pp. 1292-1302, 2004.

[2] F. Zhang and N. E. Leonard, "Cooperative control and filtering for cooperative exploration," IEEE Transactions on Automatic Control, vol. 55 , no. 3, pp. 650-663, 2010.

[3] R. Graham and J. Cortes, "Cooperative adaptive sampling via approximate entropy maximization," in Proc. of the 48st IEEE Conf. on Decision and Control, Shanghai, China, 2009, pp. 7055-7060.

[4] Z. Jin and A. L. Bertozzi, "Environmental boundary tracking and estimation using multiple autonomous vehicles," in Proc. of the 46th IEEE Conf. on Decision and Control, New Orleans, LA, 2008, pp. 4918-4923.

[5] C. Torney, Z. Neufeld, and L. Couzin, "Context-dependent interaction leads to emergent search behavior in social aggregates," Proceedings of the National Academy of Sciences, vol. 106, no. 52, pp. 22 055$22060,2009$.

[6] C. Baldassano and N. E. Leonard, "Explore vs. exploit: Task allocation for multi-robot foraging," 2009.

[7] D. Baronov and J. Baillieul, "Search decisions for teams of automata," in Proc. of the 47st IEEE Conf. on Decision and Control, Cancun, Mexico, 2008, pp. 1133-1137.

[8] K. Lerman, C. Jones, Galstyan, and M. J. Mataric, "Analysis of dynamic task allocation in multi-robot systems," International Journal of Robotics Research, vol. 25, no. 3, pp. 225-241, 2006.

[9] J. L. Ny, M. M. Zavlan, and G. J. Pappas, "Resource allocation for signal detection with active sensors," in Proc. of the 48st IEEE Conf. on Decision and Control, Shanghai, China, 2009, pp. 8561-8566.

[10] K. Gu, V. L. Kharitonov, and J. Chen, Stability of Time-Delay Systems. Boston: Birkhäuser, 2003.

[11] S. Elaydi and S. Zhang, "Stability and periodicity of difference equations with finite delay," Funkcialaj Ekvacioj, vol. 37, pp. 401413, 1994.

[12] R. Bachmayer and N. E. Leonard, "Vehicle networks for gradient descent in a sampled environment," in Proc. of 41st IEEE Conf. on Decision and Control. IEEE, 2002, pp. 113-117.

[13] D. Simon, Optimal State Estimation. Hoboken, New Jersey: WileyInterscience, 2006.

[14] K. M. Nagpal and P. P. Khargonekar, "Filtering and smoothing in a $H_{\infty}$ setting," IEEE Transactions on Automatic Control, vol. 36, pp. 152-166, 1991.

[15] I. Yaesh and Y. Theodor, " $H_{\infty}$ optimal estimation: a turtorial," in Proc. of the 31st IEEE Conf. on Decision and Control, Tucson, Arizona, 1992, pp. 2278-2286.

[16] P. Bolzern, P. Colaneri, and G. D. Nicolao, "Transient and asymptotic analysis of discrete-time $H_{\infty}$ filters," European Journal of Control, vol. 3, pp. 317-324, 1997.

[17] P. Bolzern and M. Maroni, "New conditions for the convergence of $H_{\infty}$ filters and predictors," IEEE Transactions on Automatic Control, vol. 44, pp. 1564-1568, 1999.

[18] H. C. Berg, E. Coli in motion. New York: Springer, 2004.

[19] M. Ji and M. Egerstedt, "Distributed coordination control of multiagent systems while preserving connectedness," IEEE Transactions on Robotics, vol. 23, no. 4, pp. 693-703, 2007.

[20] F. Zhang, E. Fiorelli, and N. E. Leonard, "Exploring scalar fields using multiple sensor platforms: Tracking level curves," in Proc. 46th IEEE Conference on Decision and Control, New Orleans, LA, 2007, pp. 3579-3584.

[21] I. Yaesh and U. Shaked, "A transfer function approach to the problem of discrete-time systems: $H_{\infty}$-optimal linear control and filtering," IEEE Transactions on Automatic Control, vol. 36, pp. 1264-1271, 1991

[22] Y. Theodor, U. Shaked, and C. Souza, "A game theory approach to robust discrete-time $H_{\infty}$ estimation," IEEE Transactions on Signal Processing, vol. 42, no. 6, pp. 1486-1495, 1994.

[23] N. J. Higham and H. Kim, "Numerical analysis of a quadratic matrix equation," IMA Journal of Numerical Analysis, vol. 20, pp. 499-519, 2000.

[24] F. Zhang, M. Goldgeier, and P. S. Krishnaprasad, "Control of small formations using shape coordinates," in Proc. of 2003 International Conf. of Robotics and Automation. Taipei, Taiwan: IEEE, 2003, pp. 2510-2515. 